\title{
Lecanicillium cauligalbarum sp. nov. (Cordycipitaceae, Hypocreales), a novel fungus isolated from a stemborer in the Yao Ren National Forest Mountain Park, Guizhou
}

\author{
Ye-Ming Zhou',2, Jun-Rui Zhi', Mao Ye', \\ Zhi-Yuan Zhang', Wen-Bo Yue', Xiao Zou ${ }^{2}$
}

I The Provincial Key Laboratory for Agricultural Pest Management of the Mountainous Region, Institute of Entomology, Guizhou University, Guiyang 550025, Guizhou, China 2 Institute of Fungus Resources, Guizhou University, Guiyang, Guizhou 550025, China

Corresponding authors: Jun-Rui Zhi (jrzhi@gzu.edu.cn); Xiao-Zou (xzou@gzu.edu.cn)

Academic editor: T. Lumbsch | Received 30 September 2018 | Accepted 8 November 2018 | Published 4 December 2018

Citation: Zhou Y-M, Zhi J-R, Ye M, Zhang Z-Y, Yue W-B, Zou X (2018) Lecanicillium cauligalbarum sp. nov. (Cordycipitaceae, Hypocreales), a novel fungus isolated from a stemborer in the Yao Ren National Forest Mountain Park, Guizhou. MycoKeys 43: 59-74. https://doi.org/10.3897/mycokeys.43.30203

\begin{abstract}
A new species of entomopathogenic fungi, Lecanicillium cauligalbarum, was discovered from a survey of invertebrate-associated fungi in the Yao Ren National Forest Mountain Park in China. The synnemata of this species emerged from the corpse of a stemborer (Lepidoptera), which was hidden amongst pieces of wood on the forest floor. It differs from morphologically similar Lecanicillium species mainly in its short conidiogenous cells and ellipsoid to ovoid and aseptate conidia. Phylogenetic analysis of a combined data set comprising ITS, SSU, LSU, TEF, RPB1 and RPB2 sequence data supported the inclusion of L. cauligalbarum in the Lecanicillium genus and its recognition as a distinct species.
\end{abstract}

\section{Keywords}

Entomopathogenic fungi, Lecanicillium, multiple genes, phylogeny, new species

\section{Introduction}

The entomopathogenic fungal genus Lecanicillium W. Gams \& Zare belongs to Ophiocordycipitaceae. It is typified by Lecanicillium lecanii with Torrubiella confragosa as the sexual morph (Zare and Gams 2001, Wijayawardene et al. 2017). Lecanicillium lecanii

Copyright Ye-Ming Zhou et al. This is an open access article distributed under the terms of the Creative Commons Attribution License (CC BY 4.0), which permits unrestricted use, distribution, and reproduction in any medium, provided the original author and source are credited. 
was first named as Cephalosporium lecanii Zimm. by Zimmermann in 1898. Viegas incorporated the species in Verticillium Nees in 1939 (Gams and Zare 2001). The genus Verticillium has a wide host range, including arthropods, nematodes, plants and fungi (Goettel et al. 2008). Zare and Gams (2001) recircumscribed the genus following analyses of morphological data and sequence data for the internal transcribed spacer (ITS) rDNA region (which comprises the ITS1 spacer, $5.8 \mathrm{~S}$ coding region and ITS2 spacer). All insect pathogens formerly included in Verticillium were reclassified in a newly established genus, Lecanicillium. In more recent studies, a multilocus nuclear DNA dataset combining sequence data for the nuclear small subunit rDNA (SSU), nuclear large subunit rDNA ( $L S U)$, translation elongation factor $1 \alpha(T E F)$, DNAdependent RNA polymerase II largest subunit (RPBI) and DNA-dependent RNA polymerase II second largest subunit (RPB2) genes suggests that the genus Lecanicillium is paraphyletic (Sung et al. 2007). Phylogenetic analysis of ITS sequence data also supports this conclusion (Sukarno et al. 2009). Kepler et al. (2017) revisited the taxonomic affinities of the Cordycipitaceae (Hypocreales) and proposed that Lecanicillium should be rejected because $L$. lecanii is included within the Akanthomyces clade and the name Akanthomyces Lebert has nomenclatural priority over Lecanicillium (Kepler et al. 2017). However, Kepler et al. (2017) transferred to Akanthomyces only several species for which sufficient information was available. The phylogenetic affinities of the majority of species in the original circumscription of Lecanicillium remain uncertain. Given that there remain unresolved phylogenetic and taxonomic matters concerning Lecanicillium, Huang et al. (2018) and Crous et al. (2018) chose to describe new taxa in Lecanicillium to avoid creating further confusion in the taxonomy (Crous et al. 2018; Huang et al. 2018).

Presently, 29 Lecanicillium species have been formally described and are listed in the Index Fungorum (http://www.indexfungorum.org). Zare and Gams (2001) recognised 14 Lecanicillium species based primarily on morphology and ITS sequence data (Zare and Gams 2001). Subsequently, an additional five new Lecanicillium species, based on ITS sequence data, were described (Kope and Leal 2006, Sukarno et al. 2009, Kaifuchi et al. 2013). In order to add more sequence information with ITS, Zare and Gams (2008) reassessed the genus Verticillium and transferred four species to Lecanicillium based on ITS and SSU sequence data (Zare and Gams 2008). Except for the SSU and ITS gene, more and more researchers have labelled the Lecanicillium genus by TEF gene. Based on this, two new Lecanicillium species were confirmed based on combined with ITS and TEF sequence data (Crous et al. 2018). With combined multigene identification of species gradually becoming the convention, two new Lecanicillium species were identified based on multilocus (TEF, RPB1, RPB2, LSU and SSU) sequence data (Park et al. 2016, Chen et al. 2017). Lecanicillium sabanense was identified based on phylogenetic analysis of combined multilocus and ITS sequences (Chiriví-Salomón et al. 2015). Lecanicillium subprimulinum was identified based on combined analysis of LSU, SSU, TEF and ITS sequence data (Huang et al. 2018).

We carried out a survey of invertebrate-associated fungi in the Yao Ren National Forest Mountain Park near Sandu county in Guizhou province, China. A parasitic 
fungus was found on a stemborer (Lepidoptera) hiding amongst pieces of wood. Attempting to identify the fungus, we determined it to be a member of Lecanicillium but its morphological traits and gene sequences did not correspond with those of any known Lecanicillium species. On the basis of its morphology and molecular phylogenetic analysis of multilocus nuclear genes (TEF, RPB1, RPB2, LSU and SSU) and ITS sequence data, this fungus was suggested to be an unnamed species of Lecanicillium and is here described and named Lecanicillium cauligalbarum sp. nov.

\section{Materials and methods}

\section{Specimen collection and fungus isolation}

The specimen was collected from Yao Ren National Forest Mountain Park, Sandu county, Guizhou, China $\left(107^{\circ} 53^{\prime}, 107^{\circ} 58^{\prime} \mathrm{E} ; 24^{\circ} 54^{\prime}, 25^{\circ} 59^{\prime} \mathrm{N}\right.$, approximately $560-1365 \mathrm{~m}$ above sea level), in September 2015 by Yeming Zhou and Xiao Zou. The synnemata of this species emerged from a dead stemborer (Lepidoptera) hidden amongst pieces of wood on the forest floor. The specimen GZUIFR-2015ZHJ and two isolated strains of the fungal asexual stage, GZUIFRZHJ01 and GZUIFRZHJ02, were deposited at the Institute of Fungal Resources of Guizhou University (GZUIFR). The fungal strains were isolated on potato dextrose agar (PDA) medium; one strain was isolated from part of the body and the second strain was isolated from the synnemata.

\section{Strain culture and identification}

The isolated strains were inoculated on PDA at $25^{\circ} \mathrm{C}$ for $14 \mathrm{~d}$ under $12-\mathrm{h}$ light/12-h dark conditions. The fresh hyphae were observed with an optical microscope (OM, BK5000, OPTEC, USA) following pretreatment with lactophenol cotton blue solution or normal saline.

\section{DNA extraction, PCR amplification and sequencing}

Genomic DNA was extracted using a previously described method (Chiriví-Salomón et al. 2015, Zou et al. 2016). The primers used for PCR amplification of the ITS region, SSU, LSU, TEF, RPB1 and RPB2 are listed in Table 1 . The PCR reaction conditions employed for each genetic region followed those used in the references listed in Table 1.

To conduct phylogenetic analysis of the sequences obtained, sequences for selected taxa based on recent phylogenetic studies of Lecanicillium (Chen et al. 2017, Huang et al. 2018) and Cordycipitaceae (Sung et al. 2007, Kepler et al. 2017, Mongkolsamrit et al. 2018) were downloaded from the National Center for Biotechnology Infor- 
Table I. Primer information and provenance in this study.

\begin{tabular}{|c|c|c|}
\hline Gene & Primer & Provenance \\
\hline \multirow[t]{2}{*}{ ITS } & F: 5'-TCCGTAGGTGAACCTGCGG-3' & \multirow[t]{2}{*}{ White et al. 1990} \\
\hline & R: 5'-TCCTCCGCTTATTGATATGC-3' & \\
\hline \multirow[t]{2}{*}{$S S U$} & F: GTAGTCATATGCTTGTCTC & \multirow[t]{2}{*}{ White et al. 1990} \\
\hline & R: CTTCCGTCAATTCCTTTAAG & \\
\hline \multirow[t]{2}{*}{$L S U$} & F: GTTTCCGTAGGTGAACCTGC & \multirow[t]{2}{*}{ Curran et al. 1994} \\
\hline & R: ATATGCTTAAGTTCAGCGGGT & \\
\hline \multirow[t]{2}{*}{$T E F$} & F: 5'-GCCCCCGGCCATCGTGACTTCAT-3' & \multirow[t]{2}{*}{ van den Brink et al. 2011} \\
\hline & R: 5'-ATGACACCGACAGCGACGGTCTG-3' & \\
\hline \multirow[t]{2}{*}{$R P B 1$} & F: 5'-CCWGGYTTYATCAAGAARGT-3' & \multirow[t]{2}{*}{ Castlebury et al. 2004} \\
\hline & R: 5'-CAYCCWGGYTTYATCAAGAA-3' & \\
\hline \multirow[t]{2}{*}{ RPB2 } & F: 5'-GACGACCGTG ATCACTTTGG-3' & \multirow[t]{2}{*}{ van den Brink et al. 2011} \\
\hline & R: 5'-CCCATGGCCTGTTTGCCCAT-3' & \\
\hline
\end{tabular}

mation GenBank database (https://www.ncbi.nlm.nih.gov/genbank/). A total of 79 accessions of Cordycipitaceae were selected for this study. The sequences used in the study are listed in Table 2.

\section{Sequence alignment and phylogenetic analyses}

The DNA sequences used in this study were edited using the LASERGENE software (version 6.0; DNASTAR, Madison, WI, USA). Multiple sequence alignments for TEF, RPB1 and RPB2 were performed in MAFFT (Katoh and Standley 2013) with the default settings. Multiple sequence alignments for ITS, $L S U$ and SSU were conducted using MUSCLE algorithm (Edgar 2004) from MEGA 6 (Tamura et al. 2013). The sequences were edited manually. A multiple alignment of the combined partial ITS $+S S U+L S U+T E F+R P B 1+R P B 2$ sequences were assembled with MEGA 6 (Tamura et al. 2013) and SEQUENCEMATRIX 1.7.8 (Vaidya et al. 2011). The command 'hompart' in PAUP* 4.0b10 was used for assessment of concordance amongst the genes and the ITS region (Swofford 2001). Bayesian inference (BI) was performed using MRBAYES 3.2 (Ronquist et al. 2012) and maximum likelihood (ML) analysis was performed using RAxML (Alexandros 2014) to analyse the combined data which were divided into twelve separate partitions (Kepler et al. 2017; Mongkolsamrit et al. 2018). Two maximum likelihood (ML) analysis and Bayesian inference (BI) analysis were performed. The first analysis was performed as reported by Huang et al. (2018), using the Simplicillium lanosoniveum as the outgroup. The second analysis was performed with Akanthomyces, Samsoniella, Blackwellomyces, Hevansia, Simplicillium, all the lecanicillium and use of Beauveria as outgroup (Mongkolsamrit et al. 2018). Nucleotide substitution models were determined by MrModeltest 2.3 (Nylander 2004). For BI, 10000000 generations were performed with one tree selected every 500th generation and the GTR $+\mathrm{I}+\mathrm{G}$ evolutionary model was used. For ML, the model GTRGAMMA was used and a bootstrap analysis with 500 replicates was performed to assess statistical support for the tree topology. Phylogenetic trees were viewed with TREEGRAPH. 
Table 2. Specimen information and GenBank accession numbers used in this study.

\begin{tabular}{|c|c|c|c|c|c|c|c|}
\hline Species & $\begin{array}{c}\text { Voucher } \\
\text { Information }\end{array}$ & ITS & $S S U$ & $L S U$ & $T E F$ & $R P B 1$ & $R P B 2$ \\
\hline Akanthomyces waltergamsii & TBRC 7250 & MF140749 & & MF140715 & MF140835 & & \\
\hline A. waltergamsii & TBRC 7251 & MF140747 & & MF140713 & MF140833 & MF140781 & MF140805 \\
\hline \multirow[t]{2}{*}{ A. sulphureus } & TBRC 7248 & MF140758 & & MF140722 & MF140843 & MF140787 & MF140812 \\
\hline & TBRC 7249 & MF140757 & & MF140721 & MF140842 & MF140786 & MF140734 \\
\hline \multirow[t]{2}{*}{ A. thailandicus } & TBRC 7246 & MF140755 & & MF140719 & MF140840 & & MF140810 \\
\hline & TBRC 7245 & MF140754 & & & MF140839 & & MF140809 \\
\hline \multirow{2}{*}{ A. kanyawimiae } & TBRC 7242 & MF140751 & & MF140718 & MF140838 & MF140784 & MF140808 \\
\hline & TBRC 7244 & MF140752 & & MF140716 & MF140836 & & \\
\hline A. aculeatus & HUA 186145 & & MF416572 & MF416520 & MF416465 & & \\
\hline A. pistillariaeformis & HUA 186131 & & MF416573 & MF416521 & MF416466 & & \\
\hline A. coccidioperitheciatus & NHJ 6709 & JN049865 & EU369110 & EU369042 & EU369025 & EU369067 & EU369086 \\
\hline A. aculeatus & TS 772 & KC519371 & KC519368 & KC519370 & KC519366 & & \\
\hline A. tuberculatus & BCC16819 & & MF416600 & MF416546 & MF416490 & MF416647 & MF416490 \\
\hline Ascopolyporus villosus & ARSEF 6355 & & & AY886544 & DQ118750 & DQ127241 & \\
\hline Asc. polychrous & P.C. 546 & & & DQ118737 & DQ118745 & DQ127236 & \\
\hline Beauveria bassiana & ARSEF 1564 & HQ880761 & & & HQ880974 & HQ880833 & HQ880905 \\
\hline Bea. brongniartii & BCC 16585 & JN049867 & JF415951 & JF415967 & JF416009 & JN049885 & JF415991 \\
\hline Blackwellomyces cardinalis & OSC 93610 & JN049843 & AY184974 & AY184963 & EF469059 & EF469088 & EF469106 \\
\hline Bla. cardinalis & OSC 93609 & & AY184973 & AY184962 & DQ522325 & DQ522370 & DQ522422 \\
\hline \multirow[t]{2}{*}{ Bla. pseudomilitaris } & NBRC 101409 & JN943305 & JN941748 & JN941393 & & JN992482 & \\
\hline & NBRC 101410 & JN943307 & JN941747 & JN941394 & & JN992481 & \\
\hline Gibellula longispora & NHJ 12014 & & EU369098 & & EU369017 & EU369055 & EU369075 \\
\hline \multirow[t]{3}{*}{ Gibellula sp. } & NHJ 7859 & & EU369107 & & & EU369064 & EU369085 \\
\hline & NHJ 10788 & & EU369101 & EU369036 & EU369019 & EU369058 & EU369078 \\
\hline & NHJ 5401 & & EU369102 & & & EU369059 & EU369079 \\
\hline G. ratticaudata & ARSEF 1915 & JN049837 & DQ522562 & DQ518777 & DQ522360 & DQ522408 & DQ522467 \\
\hline Hevansia nelumboides & BCC 41864 & JN201871 & JN201863 & JN201873 & JN201867 & & \\
\hline Hev. novoguineensis & NHJ 11923 & & EU369095 & EU369032 & EU369013 & EU369052 & EU369072 \\
\hline Hev. arachnophila & NHJ 10469 & & EU369090 & EU369031 & EU369008 & EU369047 & \\
\hline Hev. cinerea & NHJ 3510 & & EU369091 & & EU369009 & EU369048 & EU369070 \\
\hline Lecanicillium acerosum & CBS418.81 & EF641893 & KM283762 & KM283786 & KM283810 & KM283832 & KM283852 \\
\hline L. antillanum & CBS350.85 & AJ292392 & AF339585 & AF339536 & DQ522350 & DQ522396 & DQ522450 \\
\hline L. aphanocladii & CBS797.84 & & KM283763 & KM283787 & KM283811 & KM283833 & KM283853 \\
\hline L. aranearum & CBS726.73a & AJ292464 & AF339586 & AF339537 & EF468781 & EF468887 & EF468934 \\
\hline L. araneicola & BTCC-F35 & AB378506 & & & & & \\
\hline L. araneogenum & GZU1031Lea & & KX845705 & KX845703 & KX845697 & KX845699 & KX845701 \\
\hline \multirow[t]{2}{*}{ L. attenuatum } & CBS402.78 & AJ292434 & AF339614 & AF339565 & EF468782 & EF468888 & EF468935 \\
\hline & KACC42493 & & KM283756 & KM283780 & KM283804 & KM283826 & KM283846 \\
\hline \multirow[t]{2}{*}{ L. cauligalbarum } & GZUIFRZHJ01 & MH730663 & MH730665 & MH730667 & MH801920 & MH801922 & MH801924 \\
\hline & GZUIFRZHJ02 & MH730664 & MH730666 & MH730668 & MH801921 & MH801923 & MH801925 \\
\hline L. dimorphum & CBS345.37 & & KM283764 & KM283788 & KM283812 & KM283834 & KM283854 \\
\hline L. flavidum & CBS300.70D & EF641877 & KM283765 & KM283789 & KM283813 & & KM283855 \\
\hline L. fungicola var. aleophilum & CBS357.80 & NR_111064 & KM283767 & KM283791 & KM283815 & KM283835 & KM283856 \\
\hline L. fungicola var: fungicola & CBS992.69 & NR_119653 & KM283768 & KM283792 & KM283816 & & KM283857 \\
\hline L. fusisporum & CBS164.70 & AJ292428 & KM283769 & KM283793 & KM283817 & KM283836 & KM283858 \\
\hline L. kalimantanense & BTCC-F23 & $\mathrm{AB} 360356$ & & & & & \\
\hline \multirow[t]{2}{*}{ L. lecanii } & CBS101247 & JN049836 & KM283770 & KM283794 & DQ522359 & KM283837 & KM283859 \\
\hline & CBS102067 & & KM283771 & KM283795 & KM283818 & KM283838 & KM283860 \\
\hline \multirow[t]{2}{*}{ L. longisporum } & CBS102072 & & KM283772 & KM283796 & KM283819 & KM283839 & KM283861 \\
\hline & CBS126.27 & & KM283773 & KM283797 & KM283820 & KM283840 & KM283862 \\
\hline L. muscarium & CBS143.62 & & KM283774 & KM283798 & KM283821 & KM283841 & KM283863 \\
\hline
\end{tabular}




\begin{tabular}{|c|c|c|c|c|c|c|c|}
\hline Species & $\begin{array}{c}\text { Voucher } \\
\text { Information }\end{array}$ & ITS & $S S U$ & $L S U$ & $T E F$ & $R P B 1$ & $R P B 2$ \\
\hline L. nodulosum & IMI 338014R & EF513012 & EF513075 & & & & \\
\hline L. pissodis & CBS118231 & & KM283775 & KM283799 & KM283822 & KM283842 & KM283864 \\
\hline \multirow[t]{2}{*}{ L. primulinum } & JCM 18525 & AB712266 & & AB712263 & & & \\
\hline & JCM 18526 & AB712267 & & AB712264 & & & \\
\hline \multirow[t]{3}{*}{ L. psalliotae } & CBS532.81 & JN049846 & AF339609 & AF339560 & EF469067 & EF469096 & EF469112 \\
\hline & CBS101270 & & EF469128 & EF469081 & EF469066 & EF469095 & $\mathrm{EF} 469113$ \\
\hline & CBS363.86 & & AF339608 & AF339559 & EF468784 & EF468890 & \\
\hline L. restrictum & CCF5252 & LT548279 & & & LT626943 & & \\
\hline L. sabanense & JCHA5 & KC633232 & KC633251 & KC875225 & KC633266 & & KC633249 \\
\hline L. saksenae & IMI 179841 & AJ292432 & & & & & \\
\hline \multirow[t]{2}{*}{ L. subprimulinum } & HKAS99548 & MG585314 & MG585316 & MG585315 & MG585317 & & \\
\hline & HKAS99549 & MG585318 & MG585320 & MG585319 & MG585321 & & \\
\hline \multirow[t]{2}{*}{ L. testudineum } & UBOCC-A112180 & LT992874 & & & LT992868 & & \\
\hline & UBOCC-A116026 & LT992871 & & & LT992867 & & \\
\hline L. tenuipes & CBS309.85 & JN036556 & KM283778 & KM283802 & DQ522341 & KM283844 & KM283866 \\
\hline \multirow[t]{2}{*}{ L. uredinophilum } & KACC44082 & & KM283758 & KM283782 & KM283806 & KM283828 & KM283848 \\
\hline & KACC47756 & & KM283759 & KM283783 & KM283807 & KM283829 & KM283849 \\
\hline L. wallacei & CBS101237 & EF641891 & AY184978 & AY184967 & EF469073 & EF469102 & EF469119 \\
\hline Samsoniella inthanonensis & TBRC 7915 & MF140761 & & MF140725 & MF140849 & MF140790 & MF140815 \\
\hline Sam. inthanonensis & TBRC 7916 & MF140760 & & MF140724 & MF140848 & MF140789 & MF140814 \\
\hline \multirow[t]{2}{*}{ Sam. aurantia } & TBRC 7271 & MF140764 & & MF140728 & MF140846 & MF140791 & MF140818 \\
\hline & TBRC 7272 & MF140763 & & MF140727 & MF140845 & & MF140817 \\
\hline \multirow[t]{2}{*}{ Sam. alboaurantium } & CBS 240.32 & AY624178 & JF415958 & JF415979 & JF416019 & JN049895 & JF415999 \\
\hline & CBS 262.58 & MH857775 & & MH869308 & JQ425685 & MF416654 & MF416448 \\
\hline Simplicillium lamellicola & CBS 116.25 & AJ292393 & AF339601 & AF339552 & DQ522356 & DQ522404 & DQ522462 \\
\hline \multirow[t]{2}{*}{ Sim. lanosoniveum } & CBS 704.86 & AJ292396 & AF339602 & AF339553 & DQ522358 & DQ522406 & DQ522464 \\
\hline & CBS 101267 & AJ292395 & AF339603 & AF339554 & DQ522357 & DQ522405 & DQ522463 \\
\hline Sim. obclavatum & CBS 311.74 & & AF339567 & AF339517 & EF468798 & & \\
\hline
\end{tabular}

\section{Results}

\section{Sequencing and phylogenetic analysis}

The first sequence dataset consisted of 3793 bases, including inserted gaps (ITS: 506 bp; SSU: 579 bp; LSU: 490 bp; TEF: 772 bp; RPB1: 561 bp; RPB2: 885 bp). The second sequence dataset consisted of 2944 bases, including inserted gaps (ITS: 526 bp; SSU: 456 bp; LSU: 409 bp; TEF: 386 bp; RPB1: 500 bp; RPB2: 667 bp). No significant differences in topology were observed between the BI and ML phylogenies. The first tree formed with almost all the Lecanicillium species (only Lecanicillium evansii could not be found in the NCBI) and one Simplicillium species (Simplicillium lanosoniveum). The phylogeny was resolved into 4 clades obviously. Lecanicillium cauligalbarum formed an independent branch in a polytomy together with a clade containing L. flavidum and L. fungicola and a major clade consisting of 27 accessions. The $L$. cauligalbarum lineage received maximum statistical support (BI posterior probabilities 1, ML boostrap 100\%), which still remains unnamed (Figure 1). In the second tree, the four Lecanicillium clades were also be supported. Lecanicillium cauligalbarum formed an independent branch in a polytomy together with a clade containing Blackwellomyces cardinalis and Blackwellomyces pseudomilitaris (BI posterior probabilities 1, ML boostrap 85\%) (Figure 2). 


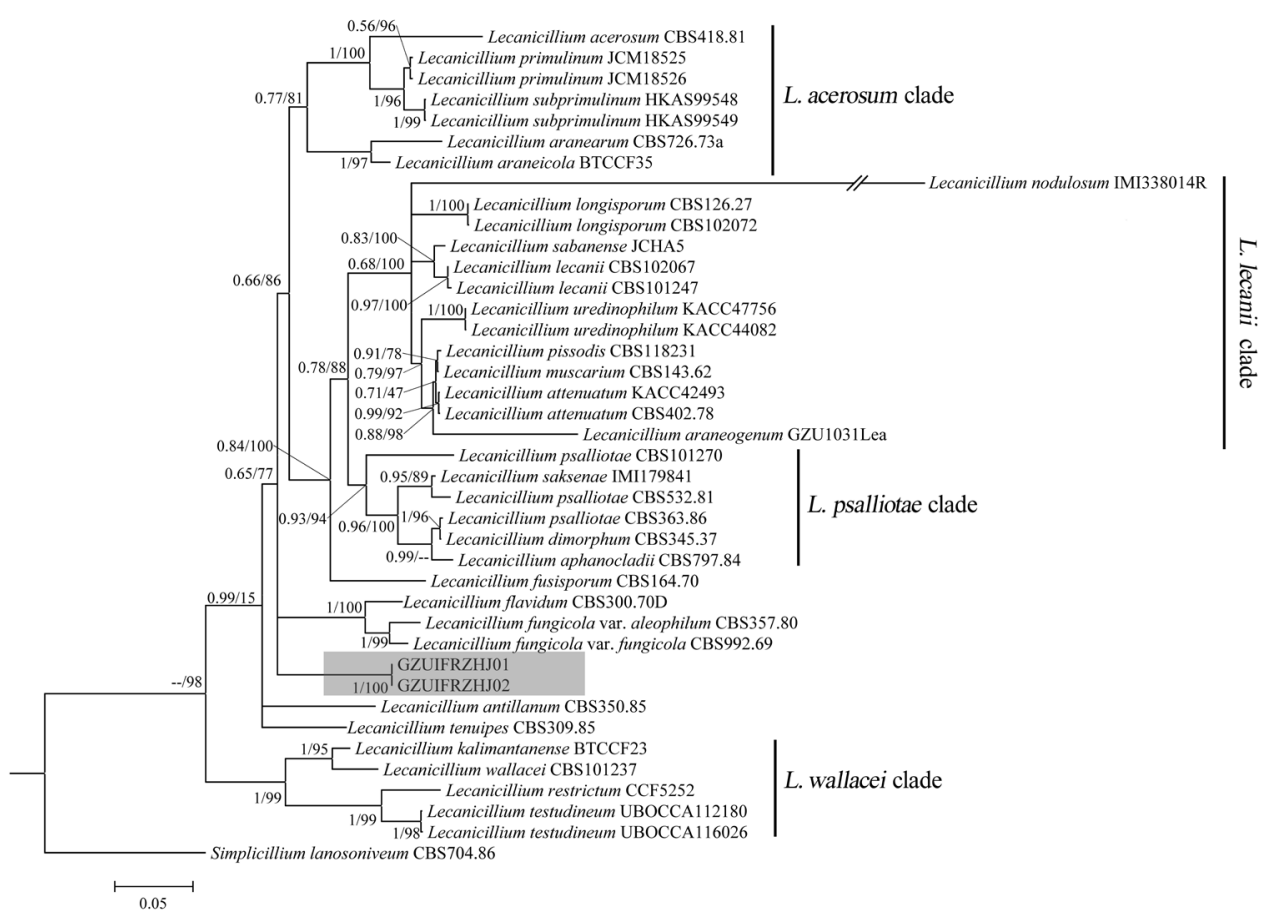

Figure I. Phylogenetic analysis of the isolated strains GZUIFRZHJ01 and GZUIFRZHJ02 and related species derived from a combined dataset of partial ITS $+S S U+L S U+T E F+R P B 1+R P B 2$ sequences. Statistical support values $(\geq 0.5 / 50 \%)$ are shown at the nodes for BI posterior probabilities/ML boostrap support.

\section{Taxonomy}

\section{Lecanicillium cauligalbarum X. Zou, J.R. Zhi \& Y.M. Zhou, sp. nov.} MycoBank: MB827984

Figure 3

Diagnosis. Characterised by phialides gradually tapering towards the apex, solitary or 2-3 whorls, 9-14.4 × 1.4-1.8 $\mu \mathrm{m}$. Conidia cylindric, aseptate, 3.6-6.3 × 0.9-1.8 $\mu \mathrm{m}$.

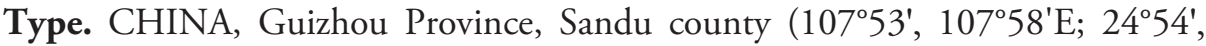
$25^{\circ} 59^{\prime} \mathrm{N}$, approximately 560-1365 $\mathrm{m}$ above sea level), September 2015, Yeming Zhou $\&$ Xiao Zou. Sequences from isolated strains (GZUIFRZHJ01 and GZUIFRZHJ02) have been deposited in GenBank (accession numbers to be provided).

Description. Colony on PDA $15 \mathrm{~mm}$ in diameter after 7 days, $33 \mathrm{~mm}$ in diameter after 14 days at $25^{\circ} \mathrm{C}$, colony circular, white, cottony, umbonate, with radiating surface texture from above, with clear radial crack and primrose-yellow from reverse. Mycelium 0.9-1.8 $\mu \mathrm{m}$ wide, hyaline, smooth, septated, branched. Conidiophores usually arising from aerial hyphae, sporulate abundant. Phialides gradually tapering towards the apex, solitary or 2-3 whorls, 9-14.4 × 1.4-1.8 $\mu \mathrm{m}$. Conidia cylindric, aseptate, $3.6-6.3 \times 0.9-1.8 \mu \mathrm{m}$. In culture, both phialides and conidia are of similar general shape and size to those found on the host stemborer. 


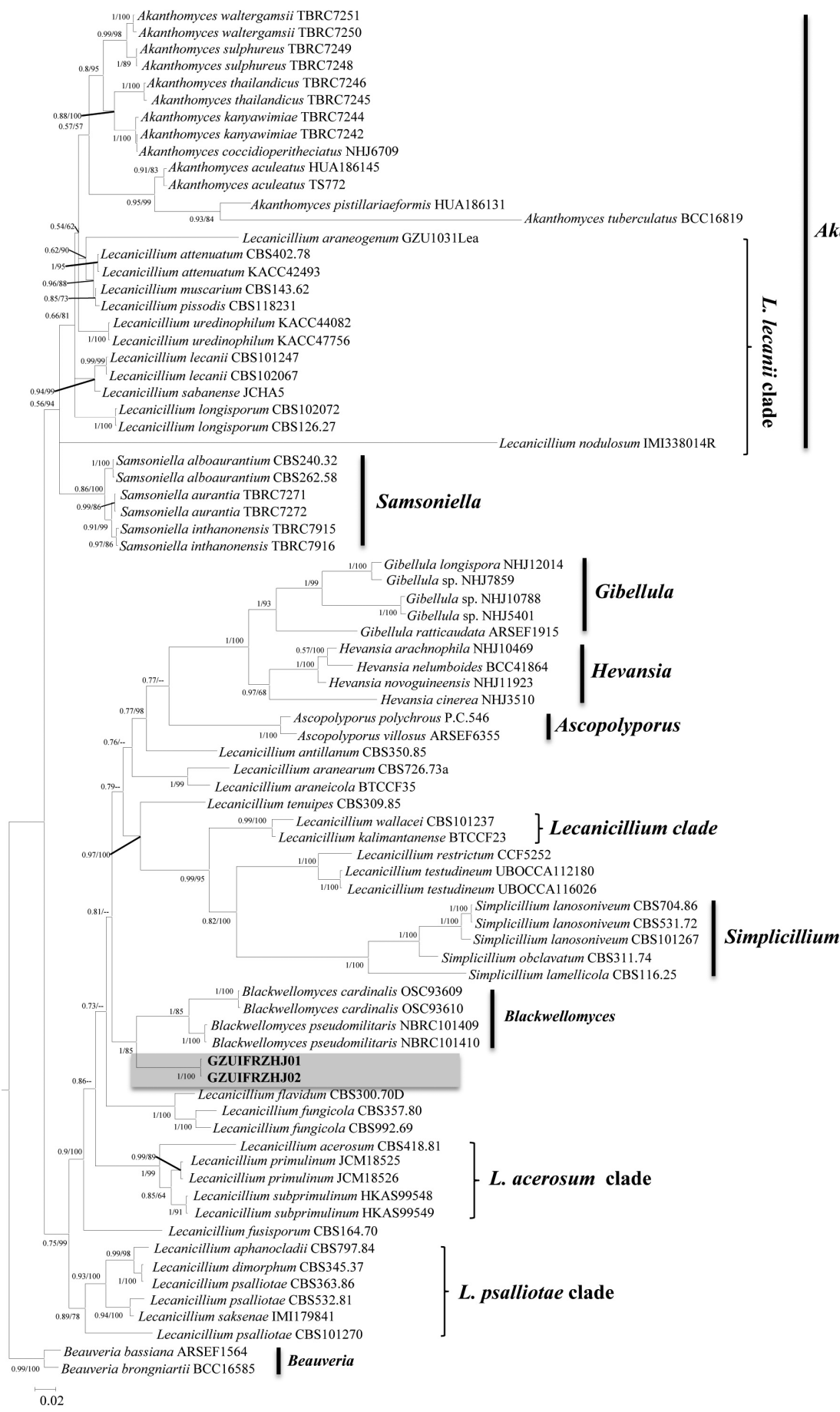

kanthomyces 

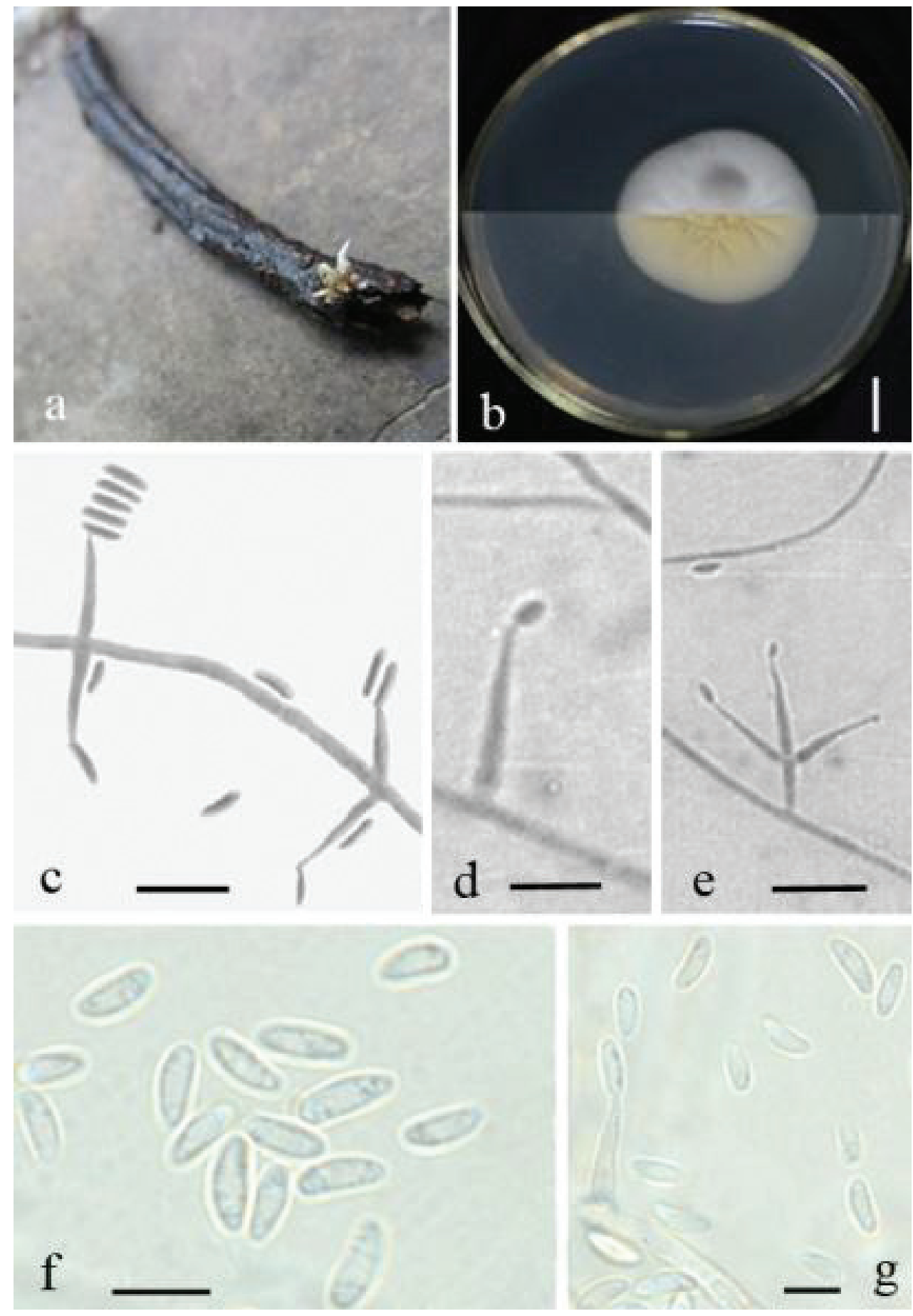

Figure 3. Lecanicillium cauligalbarum. a Synnemata emerged from the corpse of a stemborer (Lepidoptera) b Culture plate, showing the front (upper) and the back (lower) of the colony, cultured on PDA medium c-e Phialides solitary or in 2-3 whorls $\mathbf{f}-\mathbf{g}$ Conidia. Scale bars: $10 \mathrm{~mm}(\mathbf{b}, \mathbf{c}, \mathbf{e}), 5 \mu \mathrm{m}(\mathbf{d}, \mathbf{f}, \mathbf{g})$. 
Host. Stemborer (Lepidoptera) hidden amongst wooden sticks.

Habitat and distribution. Hidden amongst pieces of wood in humid forests of southwest China.

Etymology. The epithet 'cauligalbarum' refers to the host (stemborer).

Teleomorph. Not known.

Remarks. With regard to phylogenetic relationships, L. cauligalbarum is closely related to the L. fungicola clade and L. fusisporum. The two strains (GZUIFRZHJ01 and GZUIFRZHJ02) formed a distinct lineage. All Lecanicillium species were included in the phylogenetic analysis except for $L$. evansii for which sequence data could not be located in public databases, although Zare and Gams (2001) published ITS sequences. The morphological features of L. evansii include brownish-cream to brown reverse, phialides solitary or up to 3-4 per node and two types of the conidia, slightly falcate with a pointed end macroconidia 4.5-7.5 $\times 0.8-1.2 \mu \mathrm{m}$ and slightly curved microconidia 2.0-3.0 $\times 0.8-1.2 \mu \mathrm{m}$ (Zare and Gams 2001). L. evansii is distinct from $L$. cauligalbarum, which has conidia of 3.6-6.3 $\times 0.9-1.8 \mu \mathrm{m}$ and 9-14.4 × 1.4-1.8 $\mu \mathrm{m}$ phialides.

In morphology L. cauligalbarumis is similar to L. aphanocladii, L. attenuatum and L. nodulosum with regard to the short conidiogenous cell (Table 3). However, L. cauligalbarum is distinguished by the pattern of spore production and the frequency of the wheel structure.

Table 3. Morphological comparison among Lecanicillium cauligalbarum and the other related species.

\begin{tabular}{|c|c|c|c|c|}
\hline Species & Colonies & Conidiogenous cell & Conidia & Refrence \\
\hline $\begin{array}{l}\text { Lecanicillium } \\
\text { acerosum }\end{array}$ & White, yellow reverse & $\begin{array}{l}\text { Solitary or up to } 4-5,30- \\
32 \times 1.8-2.2 \mu \mathrm{m}\end{array}$ & $\begin{array}{l}\text { Macroconidia fusiform, straight to slightly } \\
\text { falcate, } 15-20 \times 1.6-2.2 \mu \mathrm{m} \text {, microconidia } \\
\text { fusiform, } 4.5-7.5 \times 1.0-1.5 \mu \mathrm{m}\end{array}$ & $\begin{array}{c}\text { Zare and } \\
\text { Gams } 2001\end{array}$ \\
\hline L. antillanum & $\begin{array}{l}\text { White, cream-coloured } \\
\text { reverse }\end{array}$ & $\begin{array}{l}\text { Solitary or up to } 6 \text {, subulate, } \\
18-31 \times 1 \mu \mathrm{m} \text { (at the top) }\end{array}$ & $\begin{array}{c}\text { Macroconidia fusiform, } 11-18 \times 0.8-1.5 \mu \mathrm{m} \text {, } \\
\text { microconidia ellipsoidal, } 3-4 \times 0.8-1.2 \mu \mathrm{m}\end{array}$ & $\begin{array}{c}\text { Zare and } \\
\text { Gams } 2001\end{array}$ \\
\hline L. aphanocladii & $\begin{array}{l}\text { White, red, reddish-white } \\
\text { to cream-coloured reverse }\end{array}$ & $\begin{array}{c}\text { Solitary, in pairs, verticillate, } \\
\text { flask-shaped in the beginning, } \\
\text { tapering into a thread-like neck, } \\
\quad 4.5-11 \times 1.0-1.8 \mu \mathrm{m}\end{array}$ & $\begin{array}{l}\text { Solitary, oval to sub-globose, } 2.7-4 \times 1.5- \\
2.2 \mu \mathrm{m}\end{array}$ & $\begin{array}{c}\text { Zare and } \\
\text { Gams } 2001\end{array}$ \\
\hline L. aranearum & $\begin{array}{l}\text { White, yellowish-cream } \\
\text { reverse }\end{array}$ & $\begin{array}{l}\text { Tapering towards the apex, } \\
20-30 \times 1.2-1.5 \mu \mathrm{m}\end{array}$ & $\begin{array}{c}\text { Straight or curved, usually asymmetrically } \\
\text { narrowed or subacute at the ends, } 5-8 \times 0.7- \\
1.5 \mu \mathrm{m}\end{array}$ & $\begin{array}{c}\text { Zare and } \\
\text { Gams } 2001\end{array}$ \\
\hline L. araneicola & $\begin{array}{l}\text { White, creamy-white } \\
\text { reverse }\end{array}$ & $\begin{array}{c}\text { Solitary or in whorls of } 2-4 \text {, } \\
\text { slender, tapering toward the tip, } \\
\text { (14-) } 19-31.5 \times 1-2 \mu \mathrm{m}\end{array}$ & $\begin{array}{l}\text { Macroconidia slightly curved to nearly } \\
\text { straight, }(7.5-) 8.5-12(-14) \times 1.5-2 \mu \mathrm{m} \text {, } \\
\text { microconidia allantoid to ellipsoidal with } \\
\text { round ends, } 3-5 \times 1-2 \mu \mathrm{m}\end{array}$ & $\begin{array}{l}\text { Sukarno et } \\
\text { al. } 2009\end{array}$ \\
\hline L. araneogenum & $\begin{array}{l}\text { White to light grey, light } \\
\text { yellow reverse }\end{array}$ & $\begin{array}{c}\text { Produced in whorls of (1-)2-6(- } \\
8), 30-64 \times 1.1-3.2 \mu \mathrm{m}\end{array}$ & $\begin{array}{l}\text { Forming mostly globose heads, cylindric, } \\
3.2-8.6 \times 1.3-1.6 \mu \mathrm{m}\end{array}$ & $\begin{array}{c}\text { Chen et al. } \\
2017\end{array}$ \\
\hline L. attenuatum & $\begin{array}{l}\text { White, yellowish-white } \\
\text { reverse }\end{array}$ & $\begin{array}{c}\text { Up to } 3-5 \text { per node, } 9-15.5 \times 1- \\
2 \mu \mathrm{m} \\
\end{array}$ & $\begin{array}{l}\text { Cylindrical with attenuate base, } 4.5- \\
6.5 \times 1.5-2.0 \mu \mathrm{m}\end{array}$ & $\begin{array}{c}\text { Zare and } \\
\text { Gams } 2001 \\
\end{array}$ \\
\hline $\begin{array}{l}\text { L. } \\
\text { cauligalbarum }\end{array}$ & $\begin{array}{l}\text { White, primrose-yellow } \\
\text { reverse }\end{array}$ & $\begin{array}{l}\text { Solitary or } 2-3 \text { whorls, } \\
9-14.4 \times 1.4-1.8 \mu \mathrm{m}\end{array}$ & Cylindrical, 3.6-6.3×0.9-1.8 $\mu \mathrm{m}$ & This work \\
\hline L. dimorphum & $\begin{array}{l}\text { White, cream to brownish- } \\
\text { cream, red reverse }\end{array}$ & $\begin{array}{l}\text { Two kinds: solitary or } 4-5 \\
\text { whorls, } 14-30 \times 1.0-1.5 \mu \mathrm{m} \text {; } \\
\text { short, } 5-12 \times 0.7-1.5 \mu \mathrm{m}\end{array}$ & $\begin{array}{l}\text { Macroconidia falcate with sharply pointed } \\
\text { ends, usually evenly curved, } 6-11 \times 1.5- \\
2.5 \mu \mathrm{m} \text {, microconidia oval to ellipsoidal, } \\
2.5-4.5 \times 1.0-1.5 \mu \mathrm{m}\end{array}$ & $\begin{array}{c}\text { Zare and } \\
\text { Gams } 2001\end{array}$ \\
\hline L. evansii & $\begin{array}{l}\text { White, creamy, brownish- } \\
\text { cream to brown reverse, }\end{array}$ & $\begin{array}{l}\text { Solitary or up to } 3-4 \text { per node, } \\
20-45 \times 1-1.2 \mu \mathrm{m}\end{array}$ & $\begin{array}{c}\text { Macroconidia slightly falcate, } 4.5-7.5 \times 0.8- \\
1.2 \mu \mathrm{m}, \text { microconidia ellipsoidal or curved, } \\
2.0-3.0 \times 0.8-1.2 \mu \mathrm{m}\end{array}$ & $\begin{array}{c}\text { Zare and } \\
\text { Gams } 2001\end{array}$ \\
\hline
\end{tabular}




\begin{tabular}{|c|c|c|c|c|}
\hline Species & Colonies & Conidiogenous cell & Conidia & Refrence \\
\hline L. flavidum & $\begin{array}{c}\text { Greyish-white to citron- } \\
\text { yellow, citron-yellow reverse }\end{array}$ & $\begin{array}{c}\text { In whorls, } 12-35 \times 1.5-2.5 \mu \mathrm{m} \\
0.5-1 \mu \mathrm{m} \text { at the tips }\end{array}$ & $\begin{array}{l}\text { Mostly fusiform, long-ellipsoidal to } \\
\text { almost cylindrical, slightly sickle-shaped, } \\
\qquad 4-8 \times 1.5-2 \mu \mathrm{m}\end{array}$ & $\begin{array}{c}\text { Zare and } \\
\text { Gams } 2008\end{array}$ \\
\hline $\begin{array}{l}\text { L. fungicola var. } \\
\text { aleophilum }\end{array}$ & White, reverse uncoloured & \begin{tabular}{|l|} 
Whorls of $3-10,15-30 \times 1.5-$ \\
$2.5 \mu \mathrm{m}, 0.5-1.5 \mu \mathrm{m}$ at the tips
\end{tabular} & $\begin{array}{l}\text { Oblong, fusiform, long ellipsoidal to almost } \\
\text { cylindrical, irregular size, } 4.5-8 \times 1-2.5 \mu \mathrm{m}\end{array}$ & $\begin{array}{c}\text { Zare and } \\
\text { Gams } 2008\end{array}$ \\
\hline $\begin{array}{l}\text { L. fungicola var. } \\
\text { fungicola }\end{array}$ & $\begin{array}{l}\text { Dirty white, reverse } \\
\text { uncoloured }\end{array}$ & $\begin{array}{l}\text { Whorls of } 3-7,14-20(- \\
45) \times 1.5-3 \mu \mathrm{m}, 0.5-1 \mu \mathrm{m} \text { at } \\
\text { the tip }\end{array}$ & \begin{tabular}{|c|} 
Fusiform, long-ellipsoidal to almost \\
cylindrical, sickle-shaped, very unequal size, \\
$4-9(-12) \times 1.5-2.5(-3.5) \mu \mathrm{m}$ \\
\end{tabular} & $\begin{array}{c}\text { Zare and } \\
\text { Gams } 2008\end{array}$ \\
\hline L. fusisporum & $\begin{array}{c}\text { White, with red reverse and } \\
\text { pigment diffusing }\end{array}$ & \begin{tabular}{|c|} 
Solitary or up to $5,16-26 \times 1.0-$ \\
$1.5 \mu \mathrm{m}$
\end{tabular} & $\begin{array}{l}\text { Fusiform, straight and rather broad, } \\
\qquad 3-5 \times 1.5-2.0 \mu \mathrm{m}\end{array}$ & \begin{tabular}{|c|} 
Zare and \\
Gams 2001 \\
\end{tabular} \\
\hline \begin{tabular}{|l|} 
L. \\
kalimantanense
\end{tabular} & $\begin{array}{l}\text { White, creamy-white } \\
\text { reverse }\end{array}$ & $\begin{array}{c}\begin{array}{c}\text { Solitary or more often in whorls } \\
\text { of } 2-5 \text {, slender, tapering toward } \\
\text { the apex, } 12.5-36 \times 1-2 \mu \mathrm{m}\end{array} \\
\end{array}$ & $\begin{array}{c}\text { Acerose to fusoid with pointed ends, slightly } \\
\text { curved, of varying size, (3.5-)4.5-12×1-2 } \\
\mu \mathrm{m}\end{array}$ & $\begin{array}{l}\text { Sukarno et } \\
\text { al. } 2009\end{array}$ \\
\hline L. lecanii & $\begin{array}{l}\text { Yellowish-white, deep } \\
\text { yellow reverse }\end{array}$ & $\begin{array}{l}\text { Aculeate and strongly tapering, } \\
\text { singly or up to } 6,11-20(- \\
30) \times 1.3-1.8 \mu \mathrm{m}\end{array}$ & $\begin{array}{c}\text { Typically short-ellipsoidal, } 2.5-3.5(-4.2) \times 1- \\
1.5 \mu \mathrm{m} \text {, homogeneous in size and shape }\end{array}$ & $\begin{array}{l}\text { Zare and } \\
\text { Gams } 2001\end{array}$ \\
\hline L. longisporum & $\begin{array}{l}\text { White to sulphur-yellow, } \\
\text { cream-coloured to pale } \\
\text { yellow reverse }\end{array}$ & $\begin{array}{c}\text { Tapering towards the apex(sub- } \\
\text { aculeate), singly or up to } 5-6 \\
\text { or on secondary phialides, } \\
20-40 \times 1.2-2.7 \mu \mathrm{m}\end{array}$ & $\begin{array}{l}\text { Produced in globose heads, el } \\
\text { oblong-oval, } 5.0-10.5 \times 1.5\end{array}$ & $\begin{array}{l}\text { Zare and } \\
\text { Gams } 2001\end{array}$ \\
\hline L. muscarium & $\begin{array}{l}\text { White, cream-co } \\
\text { uncoloured r }\end{array}$ & $\begin{array}{c}\begin{array}{c}\text { Solitary or up to } 6 \text { (less frequent } \\
\text { than in } L . \text { lecanii), }(15-) 20- \\
35 \times 1-1.5 \mu \mathrm{m}\end{array} \\
\end{array}$ & $\begin{array}{l}\text { Produced in globose heads, ellipsoidal to } \\
\text { subcylindrical, more irregular in size and } \\
\text { shape, }(2-) 2.5-5.5(-6) \times 1-1.5(-1.8) \mu \mathrm{m}\end{array}$ & $\begin{array}{l}\text { Zare and } \\
\text { Gams } 2001\end{array}$ \\
\hline L. nodulosum & $\begin{array}{l}\text { White, cream-coloured } \\
\text { reverse }\end{array}$ & $\begin{array}{l}\text { Subulate, up to } 6,10- \\
20 \times 1.5 \mu \mathrm{m}\end{array}$ & $\begin{array}{l}\text { Produced in heads of about } 10 \mu \mathrm{m} \text { diam., } \\
\text { oval, } 2.5-4.5 \times 1.2-1.5 \mu \mathrm{m}\end{array}$ & \begin{tabular}{|c|} 
Zare and \\
Gams 2001 \\
\end{tabular} \\
\hline L. pissodis & $\begin{array}{l}\text { White, ceram to yellow } \\
\text { reverse }\end{array}$ & $\begin{array}{l}\text { Solitary, up to } 3,16-(18-28)- \\
38 \times 1-2 \mu \mathrm{m}\end{array}$ & $\begin{array}{c}\text { Up to more than } 50 \text { formed in globose } \\
\text { droplets, cylindrical to oval, very variable in } \\
\text { size and shape, } 4-9.2 \times 1.6-2.4 \mu \mathrm{m}\end{array}$ & $\begin{array}{l}\text { Kope and } \\
\text { Leal } 2006\end{array}$ \\
\hline L. primulinum & $\begin{array}{c}\text { Pale yellow, yellowish- } \\
\text { brown reverse, brownish- } \\
\text { yellow pigment }\end{array}$ & $\begin{array}{c}\text { Solitary or in whorls of } 2-5 \text {, } \\
\text { tapering toward the tip, } 20-50(- \\
85) \times 0.8-1.8 \mu \mathrm{m}\end{array}$ & $\begin{array}{l}\text { Macroconidia ellipsoidal to cylindrical, } \\
5.0-9.5 \times 1.2-2.5 \mu \mathrm{m} \text {, microconidia oval to } \\
\text { ellipsoidal, } 3.0-4.8 \times 1.0-2.5 \mu \mathrm{m}\end{array}$ & $\begin{array}{l}\text { Kaifuchi } \\
\text { et al. } 2013\end{array}$ \\
\hline L. psalliotae & $\begin{array}{l}\text { White and red, reddish- } \\
\text { cream to cream-coloured } \\
\text { reverse, red to purple } \\
\text { pigment }\end{array}$ & $\begin{array}{c}\text { Aculeate, solitary or more often } \\
3-4(-6) \text { in whorls on each node, } \\
25-35 \times 1.0-1.5 \mu \mathrm{m}\end{array}$ & $\begin{array}{c}\text { Macroconidia curved, falcated, } 5-10 \times 1.2- \\
1.7 \mu \mathrm{m}, \text { microconidia oval or ellipsoidal, } \\
2.7-3.7 \times 1-1.5 \mu \mathrm{m}\end{array}$ & $\begin{array}{c}\text { Zare and } \\
\text { Gams } 2001\end{array}$ \\
\hline L. restrictum & $\begin{array}{l}\text { Yellowish-white, reverse } \\
\text { yellowish-white to pale } \\
\text { yellow }\end{array}$ & $\begin{array}{l}\text { Solitary or in whorls of } 2-5 \text {, } \\
\text { tapering toward the tip, }(12- \\
) 17-30(-36) \times 0.5-1.5 \mu \mathrm{m} \text {, } \\
0.3-0.5 \mu \mathrm{m} \text { wide on the tip }\end{array}$ & $\begin{array}{c}\text { Macroconidia fusiform or slightly falcate, } \\
(5-) 6-10(-12) \times 1-1.5 \mu \mathrm{m} \text {, microconidia } \\
\text { ovate, ellipsoidal, obovate or fusoid, } \\
\text { frequently slightly curved, } 2.5-3 \times 1-1.5 \mu \mathrm{m}\end{array}$ & $\begin{array}{l}\text { Crous et al. } \\
2018\end{array}$ \\
\hline L. sabanense & $\begin{array}{c}\text { Pale yellow to duller yellow, } \\
\text { orange reverse }\end{array}$ & $\begin{array}{c}\text { Solitary or in whorls of } 2-4, \\
13-19 \times 1.0-2.0 \mu \mathrm{m} \text {, gradually } \\
\text { tapering to } 0.5-1.0 \mu \mathrm{m}\end{array}$ & $\begin{array}{c}\text { Forming mostly globose heads, } 9-20 \mu \mathrm{m} \\
\text { diam, ellipsoidal to ovoid, } 3.5-4.5 \times 1.5-2.0 \\
\mu \mathrm{m}\end{array}$ & $\begin{array}{l}\text { Chiriví- } \\
\text { Salomón } \\
\text { et al. } 2015\end{array}$ \\
\hline L. saksenae & $\begin{array}{l}\text { White, creamy white } \\
\text { reverse }\end{array}$ & $\begin{array}{c}\text { Solitary or often in whorls of } \\
2-4 \text {, slender, tapering towards } \\
\text { the apex, } 14.5-36 \times 1.0-2.0 \mu \mathrm{m}\end{array}$ & $\begin{array}{l}\text { Macroconidia slightly curved, } 6-13 \times 1.5-2 \\
\mu \mathrm{m} \text {, microconidia ellipsoidal to fusoid } \\
\text { with round ends, nearly straight to slightly } \\
\text { curved, } 2.5-5 \times 1.5-2 \mu \mathrm{m}\end{array}$ & $\begin{array}{l}\text { Sukarno } \\
\text { et al. } 2009\end{array}$ \\
\hline $\begin{array}{l}\text { L. } \\
\text { subprimulinum }\end{array}$ & $\begin{array}{l}\text { Creamy, primrose-yellow } \\
\text { reverse }\end{array}$ & \begin{tabular}{|c|} 
Tapering towards apex, discrete, \\
solitary or up to $2-3$ per node, \\
$19-32 \times 1.5-3.5 \mu \mathrm{m}$ \\
\end{tabular} & $\begin{array}{l}\text { Ovoid to ellipsoidal, elongated, straight or } \\
\text { slightly curved, } 4-15 \times 2-6 \mu \mathrm{m}\end{array}$ & $\begin{array}{l}\text { Huang } \\
\text { et al. } 2018\end{array}$ \\
\hline L. testudineum & $\begin{array}{l}\text { White, centrally raised, } \\
\text { wrinkled, reverse pale } \\
\text { yellow to greyish-yellow }\end{array}$ & $\begin{array}{l}\text { Solitary or in whorls of } 2-4 \text {, } \\
\text { tapering toward the tip, } \\
(13-) 16-45(-53) \times 0.5-1 \mu \mathrm{m} \\
\text { (exceptionally } 80 \mu \mathrm{m} \text { long), } \\
0.5-1 \mu \mathrm{m} \text { wide on the tip }\end{array}$ & $\begin{array}{l}\text { Macroconidia fusiform or slightly falcate, } \\
3.5-6(-6.5) \times 1-1.5 \mu \mathrm{m} \text {, microconidia ovate, } \\
\text { ellipsoidal or fusoid, curved to reniform, } \\
2-3.5 \times 1-1.5 \mu \mathrm{m}\end{array}$ & $\begin{array}{l}\text { Crous et al. } \\
2018\end{array}$ \\
\hline L. tenuipes & White, reverse uncoloured & $\begin{array}{c}\text { Arising singly or in scanty } \\
\text { whorls, } 20-35(-40) \times 1.2-1.5 \mu \mathrm{m}\end{array}$ & $\begin{array}{c}\text { Microconidia ellipsoidal, straight, } 3.0-5.5(- \\
6.5) \times 1.0-1.5 \mu \mathrm{m} \text {, microconidia fusiform to } \\
\text { falcate, } 8-17 \times 1.5-1.8 \mu \mathrm{m}\end{array}$ & $\begin{array}{c}\text { Gams et al. } \\
\text { 1984; Zare } \\
\text { and Gams } \\
2001 \\
\end{array}$ \\
\hline \begin{tabular}{|l|} 
L. \\
uredinophilum
\end{tabular} & $\begin{array}{l}\text { White to cream coloured, } \\
\text { reverse cream coloured }\end{array}$ & $\begin{array}{l}\text { Produced singly or in whorls of } \\
\text { up to } 3-5,20-60 \times 1-2.5(-3) \mu \mathrm{m}\end{array}$ & Cylindric, oblong or ellipsoid, $3-9 \times 1.8-3 \mu \mathrm{m}$ & $\begin{array}{l}\text { Park et al. } \\
2016\end{array}$ \\
\hline L. wallacei & $\begin{array}{l}\text { White, cream-coloured to } \\
\text { creamish-brown reverse }\end{array}$ & $\begin{array}{l}\text { Sollitary or up to } 3-4 \text {, aculeate, } \\
(14-) 17-25(29) \times 0.7-1.2 \mu \mathrm{m}\end{array}$ & $\begin{array}{l}\text { Macroconidia, fusiform to falcate, } \\
(7.0-) 8.5-10.5(-12.5) \times 1.0-1.5 \mu \mathrm{m} \text {, } \\
\text { microconidia ellipsoidal to slightly falcate, } \\
(3.0-) 4.0-5.5(-6.5) \times 0.7-1.2 \mu \mathrm{m}\end{array}$ & $\begin{array}{l}\text { Zare and } \\
\text { Gams } \\
2001,2008\end{array}$ \\
\hline
\end{tabular}




\section{Discussion}

The genera Lecanicillium and Simplicillium belong to the Cordycipitaceae (Sung et al. 2007). The two genera are indistinguishable in morphological traits (Sung et al. 2001; Zare and Gams 2001). However, Lecanicillium and Simplicillium are clearly separated in molecular phylogenetic analyses (Kouvelis et al. 2008; Maharachchikumbura et al. 2015; Nonaka et al. 2013). As an insect pathogen, Lecanicillium spp. has potential for development as effective biological control agents against a number of plant diseases, insect pests and plant-parasitic nematodes (Goettel et al. 2008). Fifteen commercial preparations based on Lecanicillium spp. have been developed or are in the process of being developed (Faria and Wraight 2007). Kepler et al. (2017) concluded that Lecanicillium should be incorporated into Akanthomyces and formally transferred a number of Lecanicillium species. However, the compatibility of Lecanicillium was not so good in this study. Species that have been transferred to Akanthomyces were all assembled in the L. lecanii clade in the present study. The remaining species included in the present analyses were divided into multiple clades similar to those retrieved by Kepler et al. (2017). Relationships amongst Lecanicillium species thus appear to be more complicated than expected. Thus, we also prefer to describe the new taxon as a Lecanicillium species, consistent with Huang et al. (2018), owing to the uncertainty in generic boundaries.

In a comparison of all Lecanicillium species included in the present study, we were unable to identify morphological synapomorphies that characterise the phylogenetic groups. However, the species that show a close phylogenetic relationship are more similar in morphology than those that are phylogenetically distant. For example, the L. lecanii clade, which has globose heads with a higher number of conidia, are distinguishable from those clades that usually have one conidium visible at the top of the phialide in the phylogenetic tree presented here. In our phylogeny study, the node connecting $L$. antillanum and $L$. tenuipes is the basal node for the major clade. So the relationships of all of the lineages involved may change with more data or a different dataset. Therefore, more species are needed to enrich the phylogenetic study of Lecanicillium spp.

We know that Lecanicillium has a different origin into the Cordycipitaceae. We consider that the ones 'L. lecanii clade' in pig. 1 form a strong clade inside of Akanthomyces. Maybe all these should be moved to the Akanthomyces including Lecanicillium longisporum. In addition, the elimination of the genus may create more chaos considering the unsolved other clades.

Blackwellomyces Spatafora \& Luangsa-ard is diagnosed by the unique characters of the ascospore, which have irregularly spaced septa and do not disarticulate into partspores at maturity as advised by Kepler et al. (2017). It includes Blackwellomyces cardinalis and Blackwellomyces pseudomilitaris. Asexual morphs have been described as similar to species in Clonostachys, Hirsutella, Isaria and Mariannaea (Hywel-Jones 1994; Sung and Spatafora 2004). Although the new species are close to the Blackwellomyces in the phylogenetic tree, we think they are clearly distinguished from Blackwellomyces by 
the morphology. We also treat the new species as Lecanicillium considering the small sample and the unknown teleomorph.Thus, based on the present molecular phylogeny, derived from nuclear and ribosomal DNA sequence data, together with morphological evidence, a distinct new Lecanicillium species, L. cauligalbarum, is proposed.

\section{Acknowledgements}

This study was supported by the National Science Foundation of China (Project number: 31860507; 31860037), Guizhou international science and technology cooperation base (Project number: G[2016]5802), Major special projects of Guizhou tobacco company $(201603 ;$ 201712). We thank Robert McKenzie, PhD, from Liwen Bianji, Edanz Group China (www.liwenbianji.cn/ac), for editing the English text of a draft of this manuscript. We also thank Konstanze Bensch, from mycobank web site (www.mycobank.org/), for the suggested epithet of "cauligalbarum" to replace "bristletailum".

\section{References}

Alexandros S (2014) RAxML version 8: a tool for phylogenetic analysis and post-analysis of large phylogenies. Bioinformatics 30: 1312-1313. https://doi.org/10.1093/bioinformatics/btu033

Castlebury LA, Rossman AY, Sung G-H, Hyten AS, Spatafora JW (2004) Multigene phylogeny reveals new lineage for Stachybotrys chartarum, the indoor air fungus. Mycological Research 108: 864-872. https://doi.org/10.1017/S0953756204000607

Chen WH, Han YF, Liang ZQ, Jin DC (2017) Lecanicillium araneogenum sp. nov., a new araneogenous fungus. Phytotaxa 305: 29-34. https://doi.org/10.11646/phytotaxa.305.1.4

Chiriví-Salomón JS, Danies G, Restrepo S, Sanjuan T (2015) Lecanicillium sabanense sp. nov. (Cordycipitaceae) a new fungal entomopathogen of coccids. Phytotaxa 234: 63-74. https://doi.org/10.11646/phytotaxa.234.1.4

Crous PW, Wingfield MJ, Burgess TI, Hardy GESJ, Gené J, Guarro J, Baseia IG, García D, Gusmão LFP, Souza-Motta CM, Thangavel R, Adamćík S, Barili A, Barnes CW, Bezerra JDP, Bordallo JJ, Cano-Lira JF, de Oliveira RJV, Ercole E, Hubka V, Iturrieta-González I, Kubátová A, Martín MP, Moreau PA, Morte A, Ordoñez ME, Rodríguez A, Stchigel AM, Vizzini A, Abdollahzadeh J, Abreu VP, Adamčíková K, Albuquerque GMR, Alexandrova AV, Álvarez Duarte E, Armstrong-Cho C, Banniza S, Barbosa RN, Bellanger JM, Bezerra JL, Cabral TS, Caboň M, Caicedo E, Cantillo T, Carnegie AJ, Carmo LT, Castañeda-Ruiz RF, Clement CR, Čmoková A, Conceição LB, Cruz RHSF, Damm U, da Silva BDB, da Silva GA, da Silva RMF, Santiago ALCMdA, de Oliveira LF, de Souza CAF, Déniel F, Dima B, Dong G, Edwards J, Félix CR, Fournier J, Gibertoni TB, Hosaka K, Iturriaga T, Jadan M, Jany JL, Jurjević Ž, Kolařík M, Kušan I, Landell MF, Leite Cordeiro TR, Lima DX, Loizides M, Luo S, Machado AR, Madrid H, Magalhães OMC, Marinho P, Matočec N, Mešić A, Miller AN, Morozova OV, Neves RP, Nonaka K, Nováková A, Oberlies NH, Oliveira-Filho JRC, Oliveira TGL, Papp V, Pereira OL, Perrone G, Peterson SW, Pham THG, 
Raja HA (2018) Fungal Planet description sheets: 716-784. Persoonia-Molecular Phylogeny and Evolution of Fungi 40: 240-393. https://doi.org/10.3767/persoonia.2018.40.10

Curran J, Driver F, Ballard JWO, Milner RJ (1994) Phylogeny of Metarhizium : analysis of ribosomal DNA sequence data. Mycological Research 98: 547-552. https://doi.org/10.1016/ S0953-7562(09)80478-4

Edgar R (2004) MUSCLE: multiple sequence alignment with high accuracy and high throughput. Nucleic Acids Research 32: 1792-1797. https://doi.org/10.1093/nar/gkh340

Faria MR, Wraight SP (2007) Mycoinsecticides and Mycoacaricides: A comprehensive list with worldwide coverage and international classification of formulation types. Biological Control 43: 237-256. https://doi.org/10.1016/j.biocontrol.2007.08.001

Gams W, Hoog D, Samson RA (1984) The hyphomycete genus Engyodontium a link between Verticillium and Aphanocladium. Persoonia 12: 135-147.

Gams W, Zare R (2001) A revision of Verticillium sect. Prostrata. III. Generic classification ${ }^{\circ}$ ). Nova Hedwigia 72: 47-55.

Goettel MS, Koike M, Kim JJ, Aiuchi D, Shinya R, Brodeur J (2008) Potential of Lecanicillium spp. for management of insects, nematodes and plant diseases. Journal of Invertebrate Pathology 98: 256-261. https://doi.org/10.1016/j.jip.2008.01.009

Huang SK, Maharachchikumbura SSN, Jeewon R, Bhat DJ, Phookamsak R, Hyde KD, AlSadi AM, Kang JC (2018) Lecanicillium subprimulinum (Cordycipitaceae, Hypocreales), a novel species from Baoshan, Yunnan. Phytotaxa 348: 99-108.

Hywel-Jones NL (1994) Cordyceps khaoyaiensis, and C. pseudomilitaris, two new pathogens of lepidopteran larvae from Thailand. Mycological Research 98: 939-942. https://doi. org/10.1016/S0953-7562(09)80267-0

Kaifuchi S, Nonaka K, Mori M, Shiomi K, Ōmura S, Masuma R (2013) Lecanicillium primulinum, a new hyphomycete (Cordycipitaceae) from soils in the Okinawa's main island and the Bonin Islands, Japan. Mycoscience 54: 291-296. https://doi.org/10.1016/j. myc.2012.10.006

Katoh K, Asimenos G, Toh H (2009) Multiple alignment of DNA sequences with MAFFT. Methods in Molecular Biology 537. https://doi.org/10.1007/978-1-59745-251-9_3

Kepler RM, Luangsa-ard JJ, Hywel-Jones NL, Quandt CA, Sung GH, Rehner SA, Aime MC, Henkel TW, Sanjuan T, Zare R, Chen MJ, Li ZZ, Rossman AY, Spatafora JW, Shrestha B (2017) A phylogenetically-based nomenclature for Cordycipitaceae (Hypocreales). IMA Fungus 8: 335-353. https://doi.org/10.5598/imafungus.2017.08.02.08

Kope HH, Leal I (2006) A new species of Lecanicillium isolated from the white pine weevil. Mycotaxon 94: 331-340.

Kouvelis VN, Sialakouma A, Typas MA (2008) Mitochondrial gene sequences alone or combined with ITS region sequences provide firm molecular criteria for the classification of Lecanicillium species. Mycological Research 112: 829-844. https://doi.org/10.1016/j.mycres.2008.01.016

Maharachchikumbura SSN, Hyde KD, Jones EBG, McKenzie EHC, Huang S-K, AbdelWahab MA, Daranagama DA, Dayarathne M, D’souza MJ, Goonasekara ID, Hongsanan S, Jayawardena RS, Kirk PM, Konta S, Liu J-K, Liu Z-Y, Norphanphoun C, Pang K-L, Perera RH, Senanayake IC, Shang Q, Shenoy BD, Xiao Y, Bahkali AH, Kang J, Som- 
rothipol S, Suetrong S, Wen T, Xu J (2015) Towards a natural classification and backbone tree for Sordariomycetes. Fungal Diversity 72: 199-301. https://doi.org/10.1007/ s13225-015-0331-z

Mongkolsamrit S, Noisripoom W, Thanakitpipattana D, Wutikhun T, Spatafora JW, Luangsaard J (2018) Disentangling cryptic species with isaria-like morphs in cordycipitaceae. Mycologia 110: 230-257.

Nonaka K, Kaifuchi S, Ōmura S, Masuma R (2013) Five new Simplicillium species (Cordycipitaceae) from soils in Tokyo, Japan. Mycoscience 54: 42-53. https://doi.org/10.1016/j. myc.2012.07.002

Park MJ, Hong SB, Shin HD (2016) Lecanicillium uredinophilum sp. nov. associated with rust fungi from Korea. Mycotaxon 130: 997-1005. https://doi.org/10.5248/130.997

Ronquist F, Teslenko M, van der Mark P, Ayres DL, Darling A, Höhna S, Larget B, Liu L, Suchard MA, Huelsenbeck JP (2012) MrBayes 3.2: Efficient Bayesian Phylogenetic Inference and Model Choice Across a Large Model Space. Systematic Biology 61: 539-542. https://doi.org/10.1093/sysbio/sys029

Sukarno N, Kurihara Y, Park J-Y, Inaba S, Ando K, Harayama S, Ilyas M, Mangunwardoyo W, Sjamsuridzal W, Yuniarti E, Saraswati R, Widyastuti Y (2009) Lecanicillium and Verticillium species from Indonesia and Japan including three new species. Mycoscience 50: 369-379. https://doi.org/10.1007/S10267-009-0493-1

Sung GH, Spatafora JW (2004) Cordyceps cardinalis sp. nov. a new species of Cordyceps with an east Asian-eastern North American distribution. Mycologia 96: 658-666. https:/doi.org/ 10.1080/15572536.2005.11832962

Sung GH, Hywel-Jones NL, Sung JM, Luangsa-ard JJ, Shrestha B, Spatafora JW (2007) Phylogenetic classification of Cordyceps and the clavicipitaceous fungi. Studies in Mycology 57: 5-59. https://doi.org/10.3114/sim.2007.57.01

Sung GH, Spatafora JW, Zare R, Hodge KT, Gams W (2001) A revision of Verticillium sect. Prostrata. II. Phylogenetic analyses of SSU and LSU nuclear rDNA sequences from anamorphs and teleomorphs of the ClavicipitaceaeO). Nova Hedwigia 72: 311-328.

Swofford DL (2001) Paup*: Phylogenetic analysis using parsimony (and other methods) 4.0. b10. Sunderland, Massachusetts.

Tamura K, Stecher G, Peterson D, Filipski A, Kumar S (2013) MEGA6: Molecular Evolutionary Genetics Analysis Version 6.0. Molecular Biology and Evolution 30: 2725-2729. https://doi.org/10.1093/molbev/mst197

Vaidya G, Lohman DJ, Rudolf M (2011) SequenceMatrix: concatenation software for the fast assembly of multi-gene datasets with character set and codon information. Cladistics 27: 171-180. https://doi.org/10.1111/j.1096-0031.2010.00329.x

van den Brink J, Samson RA, Hagen F, Boekhout T, de Vries RP (2011) Phylogeny of the industrial relevant, thermophilic genera Myceliophthora and Corynascus. Fungal Diversity 52: 197-207. https://doi.org/10.1007/s13225-011-0107-z

White TJ, Bruns T, Lee S, Taylor J (1990) Amplification and direct sequencing of fungal ribosomal RNA genes for phylogenetics. In: Innis MA, Gelfand DH, Sninsky JJ, White TJ (Eds) PCR Protocols. Academic Press, San Diego, 315-322. https://doi.org/10.1016/ B978-0-12-372180-8.50042-1 
Wijayawardene NN, Hyde KD, Rajeshkumar KC, Hawksworth DL, Madrid H, Kirk PM, Braun U, Singh RV, Crous PW, Kukwa M, Lücking R, Kurtzman CP, Yurkov A, Haelewaters D, Aptroot A, Lumbsch HT, Timdal E, Ertz D, Etayo J, Phillips AJL, Groenewald JZ, Papizadeh M, Selbmann L, Dayarathne MC, Weerakoon G, Jones EBG, Suetrong S, Tian Q, Castañeda-Ruiz RF, Bahkali AH, Pang KL, Tanaka K, Dai DQ, Sakayaroj J, Hujslová M, Lombard L, Shenoy BD, Suija A, Maharachchikumbura SSN, Thambugala KM, Wanasinghe DN, Sharma BO, Gaikwad S, Pandit G, Zucconi L, Onofri S, Egidi E, Raja HA, Kodsueb R, Cáceres MES, Pérez-Ortega S, Fiuza PO, Monteiro JS, Vasilyeva LN, Shivas RG, Prieto M, Wedin M, Olariaga I, Lateef AA, Agrawal Y, Fazeli SAS, Amoozegar MA, Zhao GZ, Pfliegler WP, Sharma G, Oset M, Abdel-Wahab MA, Takamatsu S, Bensch K, de Silva NI, De Kesel A, Karunarathna A, Boonmee S, Pfister DH, Lu YZ, Luo ZL, Boonyuen N, Daranagama DA, Senanayake IC, Jayasiri SC, Samarakoon MC, Zeng X-Y, Doilom M, Quijada L, Rampadarath S, Heredia G, Dissanayake AJ, Jayawardana RS, Perera RH, Tang LZ, Phukhamsakda C, Hernández-Restrepo M, Ma X, Tibpromma S, Gusmao LFP, Weerahewa D, Karunarathna SC (2017) Notes for genera: Ascomycota. Fungal Diversity 86: 1-594. https://doi.org/10.1007/s13225-017-0386-0

Zare R, Gams W (2001) The genera Lecanicillium and Simplicillium gen. nov. Nova Hedwigia 73: $1-50$.

Zare R, Gams W (2008) A revision of the Verticillium fungicola species complex and its affinity with the genus Lecanicillium. Mycological Research 112: 811-824. https://doi. org/10.1016/j.mycres.2008.01.019

Zou X, Zhou YM, Liang ZQ, Xu FL (2016) A new species of the genus Hirsutella with helical twist neck of phialides parasitized on Tortricidae. Mycosystema 35: 807-813. 